\title{
PERFORMANCE ASSESSMENT USING ACM IN DVB-S2/ADVANCED DVB-RCS SATELLITE SYSTEMS
}

\author{
V. Boussemart*, H. Brandt ${ }^{\dagger}$ \\ German Aerospace Center (DLR e.V.) \\ Institute for Communication and Navigation, Oberpfaffenhofen, 82234 Wessling, Germany. \\ *vincent.boussemart@dlr.de ${ }^{\dagger}$ hartmut.brandt@dlr.de
}

Keywords: Simulator, ACM, DVB-S2, Advanced DVBRCS, GSE.

\begin{abstract}
This paper presents the benefits of using adaptive coding and modulation in DVB-S2/advanced DVB-RCS satellite systems. The results are based on a satellite system simulator which was developed during an ESA study. Such a simulator aims at making performance assessments before implementing a system, at developing and evaluating new algorithms like schedulers, encapsulators or resource managers. The simulator is capable of reproducing DVB-S2 and advanced DVB-RCS standards and techniques so that system performance can be analysed and optimized without the need of using a real satellite system.
\end{abstract}

\section{Introduction}

The simulation scenarios are based on a satellite using Kaband and covering Europe with several beams. The satellite system architecture can be either transparent or regenerative. In the transparent case the traffic and the signalling is processed by gateway(s) since the satellite relays the signal after having transposed it in frequency and amplified it. In the regenerative case the signal is processed on-board and forwarded to the beam(s) of interest, i.e. to the beam(s) to which the data content is destined to [1].

Since the Ka-frequency band is considered the signal is particularly subject to atmospheric effects [1, 2]. A preprocessing tool was implemented using Matlab to produce signal-to-noise and interference ratio time series taking into account spatially correlated rain attenuation, scintillation and channel interferences (mainly co-channel interference). Fade mitigation techniques (FMT) based on adaptive coding and modulation (ACM) are implemented in order to countermeasure bad channel conditions. ACM consists in using physical layer adaptation algorithms for both forwardand return-links so that the best modulation and coding scheme (ModCod) is selected according to the estimated channel state [3, 4].

The system scenarios consider a multi-beam satellite system using Ka-band for both feeder and user links. DVB-S2 is supported in the forward link [5] and DVB-RCS with ACM in the return/up link [6]. The scenarios assume traffic based on realistic ground distribution and using various services (web browsing, email, voice over IP, audio / video streaming and video conferencing). The traffic manages QoS classes and represents different terminal types and service level agreements. The simulator allows the utilization of different scheduler algorithms, encapsulation schemes, burst lengths and formats, congestion control mechanisms (detection and resolution) and an implementation of a call admission control scheme [7]. The resource allocation on the return-link is implemented using the standard terminal-burst-time-plan, signalling methods, burst formats [6].

Simulations were performed in order to assess the performance of ACM in DVB-S2 / advanced DVB-RCS satellite systems. A reference scenario was defined so that DVB-S2 using constant coding and modulation (broadcast profile) and DVB-RCS (without ACM) system was compared to the one using ACM. Measurements were gathered along the simulations and results were post-processed to reveal the benefit of utilizing ACM together with a combination of encapsulation scheme/scheduling algorithm. The simulations show that a total bandwidth gain of about $50 \%$ is possible for the forward link, using ACM and advanced encapsulation techniques like GSE (generic stream encapsulation), while the gain reaches up to $100 \%$ in the return link when using ACM, GSE and a fixed physical burst size.

The paper presents the results of the measurements performed with this complete advanced simulator system for satellite with FMT and the impressive gains that can be obtained on the forward-link and on the return-link when using adaptive coding and modulation. The paper is divided into two parts: in the first one the satellite system simulator is described and in the second one the results of the measurements are presented and analysed.

\section{Satellite System Simulator}

As mentioned in the introduction the satellite system simulator assumes a geostationary (GEO) satellite with a foot print including Europe and neighbouring Near-East countries. The simulator supports two main system scenarios: a system with transparent/bent-pipe satellite and a satellite system with a regenerative payload. In the first case the signal is simply 
relayed by the satellite meaning that communication between satellite terminals requires a double hop. In the second case the payload is processed on board so that inter-beam connectivity is possible: communication between satellite terminals requires one single hop [1] (Figure 1).

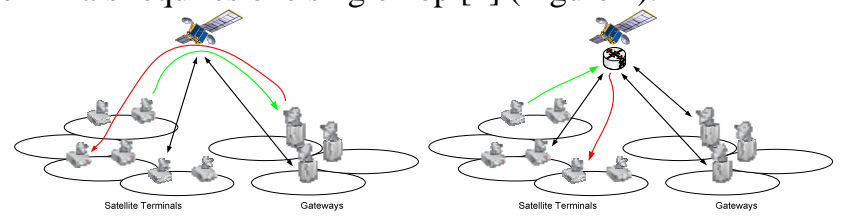

Figure 1: Transparent (left) and regenerative (right) satellite scenarios.

Both transparent and regenerative scenarios utilize Ka-band with $28 \mathrm{GHz}$ (feeder-), 30GHz (user-) up-link and $18 \mathrm{GHz}$ (feeder-), 20GHz (user-) down-link. This frequency band suffers from severe propagation effects: rain attenuation, rain depolarization and attenuation due to scintillation. These propagation impairments will cause signal-to-noise ratio (SNR) reductions [1, 2].

There are several possibilities for counteracting such propagation issues. They are called Fade Mitigation Techniques (FMT) and aim at modifying the transmission characteristics in order to keep a given level of packet error rate (PER). FMT can consist in controlling the transmission power, controlling the interference, assigning bandwidth to links, assigning transport resources, managing the quality of service (QoS), selecting appropriate and efficient MAC protocols, etc. Another FMT is based on Adaptive Coding and Modulation (ACM) and aims at selecting the most efficient modulation and coding (ModCod) mode according to the current SNR [3, 4].

The satellite system simulator is divided into three different components: the preprocessing, the simulator itself and the post-processing. The next sections describe these three components.

\subsection{Preprocessing}

The preprocessing part of the simulator is based on Matlab scripts and generates the time series and the modulation and coding (ModCod) threshold files.

The Matlab scripts are configured through an XML file which indicates several parameters such as the number of satellite terminals to be considered, the beam ID(s), the position of the

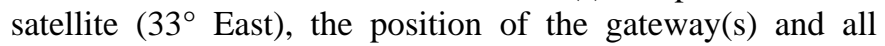
other parameters used for computing link budgets.

The coverage map of the satellite is computed and grid points are generated according to the configuration considered (spot beam patterns). The subscriber terminals are then spatially distributed in the coverage area of the satellite, i.e. in Europe and neighbouring Near-East countries.

Co-channel interferences (CCI) are computed / calculated for each grid point according to a model which depends on the traffic, the location, the time, the effect of the rain and the active users. Cross-polar interferences are not taken into account since there is no polarisation re-use. Both intermodulation and adjacent channel interferences are assumed to remain constant.

The next step of the preprocessing concerns the rain fading. The rain fading and scintillation time series are generated for each terminal link and each gateway link. The forward uplink and downlink time series and the return uplink and downlink time series for all terminals and gateways are produced from those results (Figure 2 and Figure 3). The burstiness of the time series in the return-link is related to the other interfering satellite terminals (delta of $3 \mathrm{~dB}$ ).

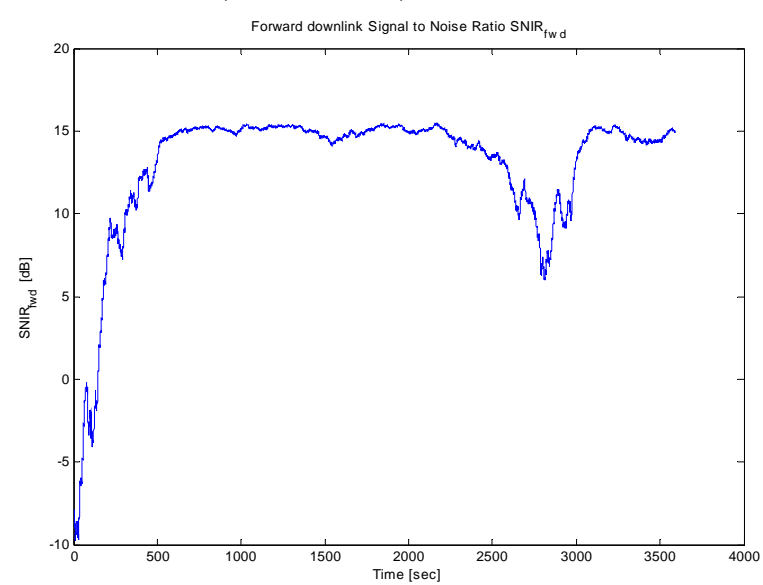

Figure 2: Example of forward downlink signal-to-noise and interference ratio time series.

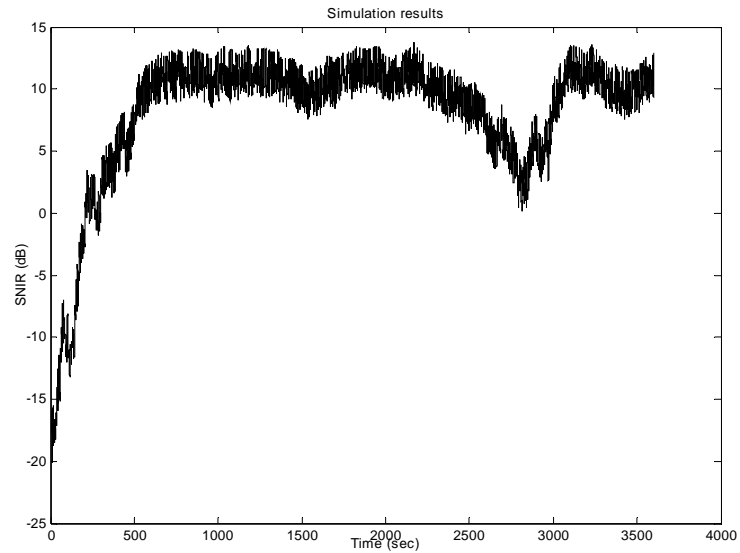

Figure 3: Example of return uplink signal-to-noise and interference ratio time series.

The last preprocessing step precalculates the FMT parameters. It generates the channel estimation parameters which are used to add channel estimation error to the input signal-to-noise ratio and to correct the measurement (errors introduced in the estimation of the channel measurement / age of the measurement because of the transmission delay). For each modulation and coding scheme a critical threshold identifies the SNR value below which the PER cannot be guaranteed (PER set to $10^{\wedge}-7$ here). Because of rain fading 
events the channel state can switch from slowly varying distortions to very rapidly varying distortions. This involves numerous modifications of the ModCod which are not acceptable. For this reason margins are introduced so that ModCod switches are more stable. ModCod thresholds (upand down-thresholds giving a hysteresis) are generated by the preprocessing so that the simulator knows, according to the current channel measurements, what is the most appropriate ModCod [8] (Figure 4).

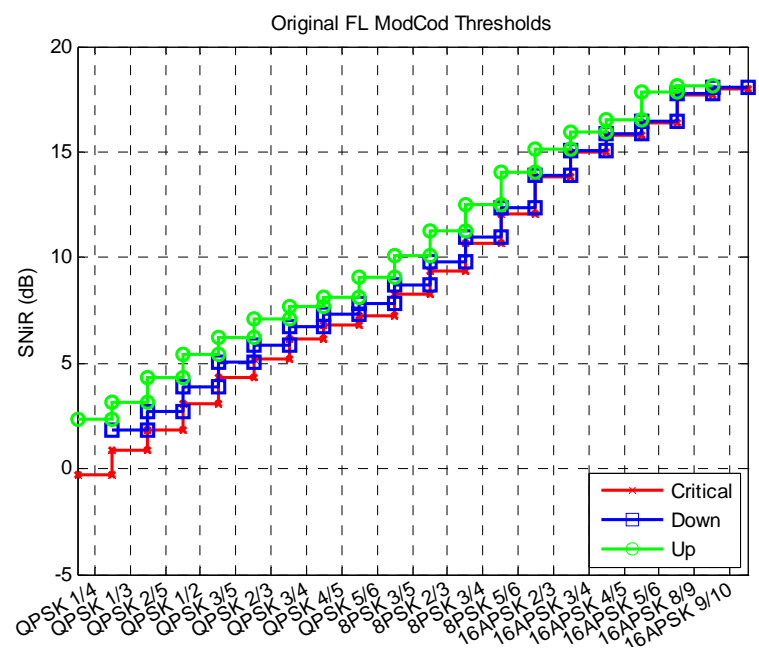

Figure 4: Example of ModCod thresholds for the FL.

\subsection{Simulator}

The simulator was developed using the object modular network testbed in $\mathrm{C}++(\mathrm{OMNeT}++)$ kernel [9]. The different components (gateway(s), satellite terminals and the satellite) are implemented as modules containing submodules (Figure 5 and Figure 6). The different layers were implemented according to the ISO reference model, i.e. from the application layer to the physical layer.

The application layer corresponds to the traffic generation. Traffic models were implemented so that the real burstiness of the Internet traffic can be reproduced [10]. The following applications are supported: web browsing (exchange of requests and responses), mail (retrieving of emails like with the post-office protocol 3, POP3), voice over IP (VoIP) with different codecs (ON/OFF model), video streaming (unidirectional reception of data) and audio/video conferencing. The different traffic models generate packets having different sizes and requirements in terms of QoS.

For sake of complexity and performance in the simulator the transport layer, more particularly the transmission control protocol (TCP), was simplified and managed by the mean of other modules: the session managers. Those are responsible of establishing and closing connections like in TCP. The size of the TCP header, for controlled transmission, and of both the real-time protocol (RTP) and user datagram protocol (UDP) were taken into account.

Packets are encapsulated according to the request for comments (RFC) which describes the internet protocol (IP).
Packets are segmented and the type of service field (ToS) is written according to the QoS. Source and destination addresses are also included.

IP packets enter into the transceiver (satellite terminal side, return-link) or into the gateway (gateway side, forward-link). They go through the interworking function (IWF) / policy enforcement point (PEP) and classifier module which takes care of routing the packets according to their QoS (different queues).

In the return-link (RL), i.e. when the packet is transmitted by the satellite terminal, the packet enters into the scheduler and stays in the buffer until slots are available. Resources on the $\mathrm{RL}$ are allocated and organized according to the terminal burst time plan (TBTP). The transmission on the RL is performed using multi-frequency - time-division multiple access (MF-TDMA). When the TBTP indicates that one or several slots are available for the current terminal the scheduler forwards the packet to the encapsulator based on a deficit round-robin algorithm. The encapsulator processes the packet according to the selected scheme and transmits it to the MAC layer. The burst is generated depending on the modulation and coding scheme attributed to the satellite terminal. The return-link is implemented following the DVBRCS standard [6].

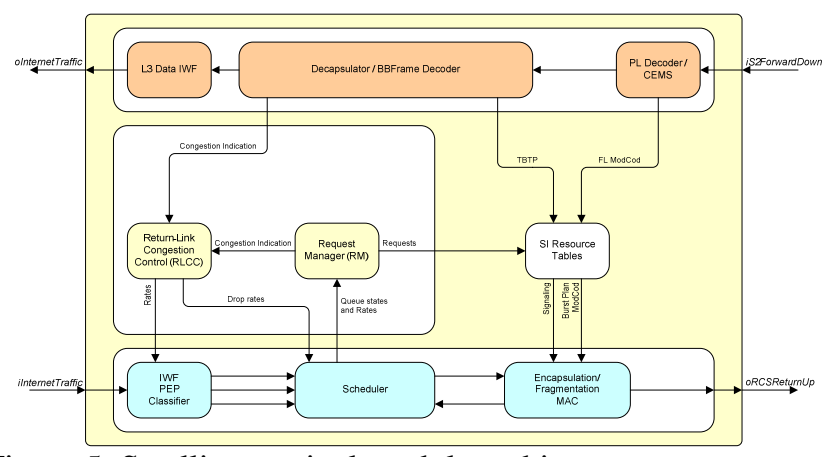

Figure 5: Satellite terminal module architecture.

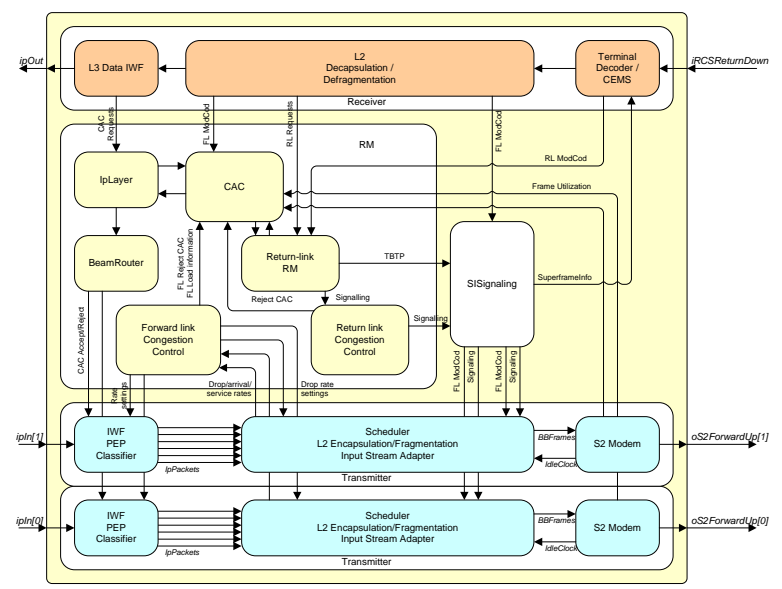

Figure 6: Gateway module architecture.

The principle remains quite the same for the forward-link (FL). The packet enters into the scheduler and stays in the queue until the scheduler gets enough resources to transmit it. 
The transmission on the FL is performed using time-division multiple access (TDMA). The scheduler is implemented based on a specific algorithm which takes care of the QoS and of the different ModCods assigned [11]. Packets, when selected, are encapsulated and forwarded to the S2 modem after being transformed into BBFRAMES according to the ModCod selected. The forward-link is implemented following the DVB-S2 standard [5].

Different mechanisms were introduced into the satellite system simulator: congestion control, call admission control (CAC) and resource management.

The congestion control mechanisms detect and resolve congestion in both forward- and return-links. The detection is performed by periodically analysing the state of the queues located into the satellite terminal and gateway schedulers. The resolution is realized by disabling CAC, by dropping packets in some queues and by regulating the traffic via the PEPs.

The call admission control (CAC) was implemented for the VoIP application. When a user performs a call, the CAC mechanism will verify that enough resources are available so that the call can be supported by the system without being interrupted. The amount of resources available is computed upon the load on both forward- and return-links. When the CAC is disabled no calls can be performed until it is switched on again.

The allocation of the resources on the return-link is realized by a specific module. Actually the satellite terminals perform constant rate assignment (CRA) when they log on so that they get a minimum amount of resources to transmit data. The satellite terminals then transmit over the time capacity requests according to the state of the queues located into their schedulers. There are two kinds of capacity requests: ratebased dynamic request (RBDC, in bps) and the volume-based dynamic request (AVBDC/VBDC, in bits). The requests are transmitted to the gateway and analysed by the return-link resource manager (RRM) module which allocates resources accordingly. The RRM module integrates different features such as the distribution of excess capacity and delay-jitter optimization.

Frames entering into the satellite terminals (forward-link, issued from the gateway) and into the gateway(s) (return-link, issued from the satellite terminals) are processed respectively by the physical layer decoder and by the terminal decoder. These two modules contain the channel estimator and ModCod selector (CEMS) module which takes care of extracting the current channel state (channel measurements), at correcting the errors introduced during the estimation (channel estimator) and at selecting the most appropriate ModCod. The next figure (Figure 7) shows an example of how the CEMS module operates. The upper graph corresponds to the carrier-to-noise ratio and the horizontal lines to the up- and down-thresholds values. The lower graph illustrates the ModCod selection according to the long fade event (time scale to the power 4).
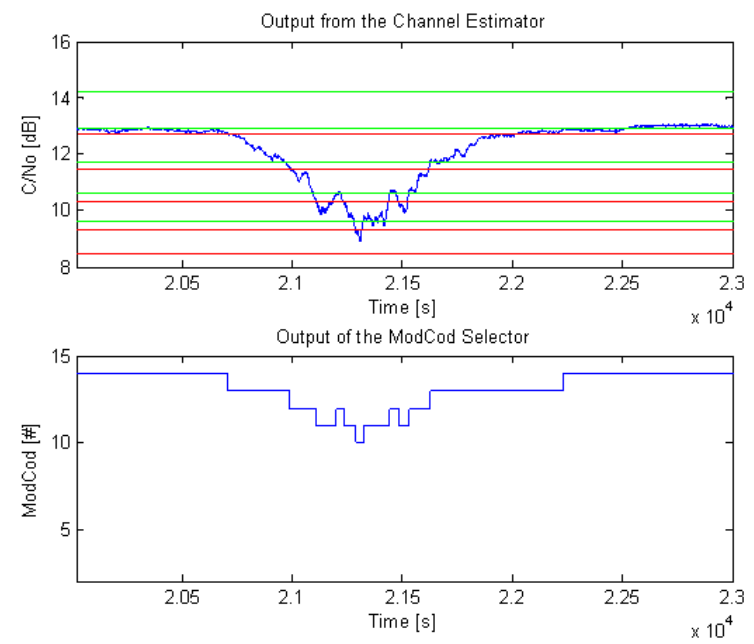

Figure 7: Example of channel estimation and ModCod selection in the simulator.

The frames received are decoded, decapsulated and IP packets are finally forwarded to the correct destination. Different encapsulation / decapsulation schemes are supported by the satellite system simulator: the Multiprotocol Encapsulation (MPE), the Unidirectional Lightweight Encapsulation (ULE) and the Generic Stream Encapsulation (GSE). For the two first ones the transportation of network layer packets is realized over MPEG-2 Transport Streams (MPEG-TS, 188 bytes).

The simulator can consider different burst lengths and formats: fixed burst length (FBL) or fixed information length (FIL). In the FBL case the number of symbols is fixed and the number of bits which can be inserted depends directly on the ModCod selected. In the FIL case the information size is fixed (in terms of bits) and the number of symbols generated is directly based on the ModCod.

\subsection{Postprocessing}

Measurement points are integrated into the different modules. These measurement points store along the simulation time the values of interest into vectors according to a time interval. The measurements can be of two kinds: aggregated or individual. Since the number of modules can become very important the measurements can be aggregated, i.e. considered among several modules that are identical (for instance all VoIP applications). Measurements can also be individual, meaning that they can be considered for one particular module (for instance the scheduler located in the first satellite terminal). At the end of the simulation measurements are available in different files.

The produced files are converted to be compliant with the software that will analyse them: Matlab. Classes were implemented into Matlab so that modules are recognized as objects. The measurements saved by the module can be accessed easily and the results can be analysed. 
Several Matlab scripts were written to get the information of interest, to perform computations and to extract statistics.

\section{Results}

The capabilities in terms of measurements and analysis are quite significant in the satellite system simulator. Thousands of parameters can be tuned so that a specific part / module of the system can be investigated. The purpose of this paper is to evaluate the impact of the utilization of adaptive coding and modulation (ACM) on both the forward-link (DVB-S2) and the return-link (DVB-RCS). To achieve this, scenarios were defined and simulations were run based on them. The description of the scenarios, the results obtained and their analysis are given in the next sections.

\subsection{Scenarios}

A reference scenario was defined such that standard systems could be compared to adaptive ones. The baseline scenario was generated by setting parameters to specific values. All congestion control and call admission control mechanisms were switched off so that they have no influence on the results.

In order to assess the performance of ACM the system was overloaded meaning that the number of satellite terminals was configured in such a way that the traffic generated exceeds the system capacity. One single beam was considered since the utilization of more beams has no significant impact on the measurements of interest. Moreover the influence of the other beams is already taken into account by the preprocessing part of the simulator (interferences). Satellite terminals are thus distributed over the beam considered, i.e. the beam ID selected.

Several assumptions were realized: the system availability was considered to be $99.7 \%$ and the coverage to be $95 \%$. The system availability corresponds to the distribution of the received SNR which is chosen so that there is a given percentage of link availability in the coverage area. Both return- and forward-links coding and modulation were fixed (CCM) respectively to QPSK 2/3 (DVB-RCS) and QPSK 3/4 (DVB-S2 broadcast profile).

The capacity of the forward-link was set to one single carrier with 19.5Msps. The number of satellite terminals was computed knowing the different traffic bandwidths and the distributions among the terminal types. Actually three satellite terminals are supported by the simulator: small-office / homeoffice (SOHO) with limited number of users (around 2 users), small- to medium-enterprise 1 (SME1, around 20 users) and small- to medium-enterprise 2 (SME2, around 50 users). For each terminal two service level agreements are considered: gold and silver. This results into six terminal types which are distributed as follows in the scenarios: $48 \%$ SOHO, $26 \%$ SME1 and 26\% SME2 with gold / silver respectively 1/3 / 2/3.
Based on the coding and modulation selected on the FL, on the capacity available and on the overhead (encapsulation, headers, etc.) the number of satellite terminals necessary to reach the congestion state (overload) was computed. The superframe (SF) configuration requested by the return-link was then realized assuming that SOHO terminals use 1Msps carriers, SME1 terminals use 2Msps carriers but can downgrade and make use of 1Msps if necessary and SME2 terminals use 4Msps carriers but can downgrade and make use of both 2Msps and 1Msps carriers if necessary. The SF duration was fixed to $200 \mathrm{~ms}$ and the number of carriers to 6 , 2 and 1 respectively with carrier capacity 1Msps, 2Msps and 4Msps (extra carriers are included for minislots). This leads to a total of 560 satellite terminals that are distributed in the coverage area of the satellite beam ID considered.

Internet terminals (INET terminals) were inserted to assure the communication between the satellite terminals and the wide area network (WAN). INET terminals are located directly behind the gateway and represent the users that are not making use of satellite terminals. Other users are directly connected to satellite terminals (Figure 8).

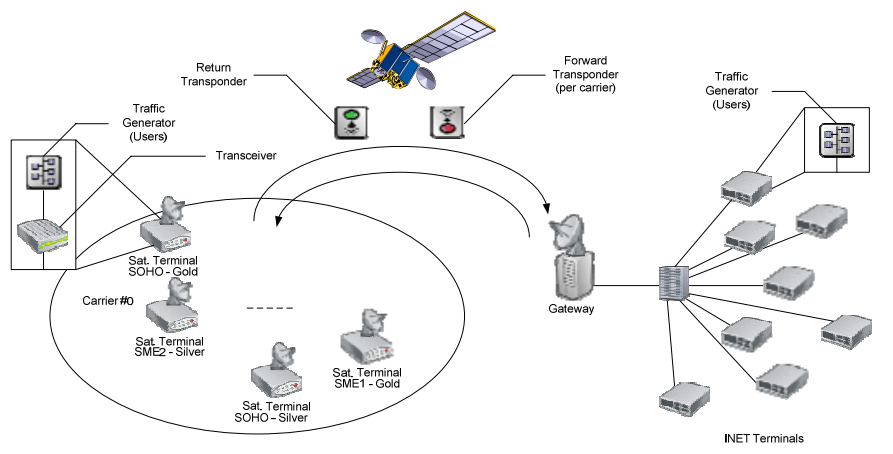

Figure 8: Overall satellite system simulator architecture.

For the baseline scenario the whole satellite system simulator was configured so that it follows as much as possible the DVB-RCS and DVB-S standards. All optimizations were switched off: constant coding and modulation (CCM) was considered, policy enforcement points (PEPs) were disabled, congestion control mechanisms were inhibited, call admission control was not selected, distribution of excess capacity and delay jitter optimization were disabled in the RRM module and a "simple" scheduler was chosen for the forward-link. The Multiprotocol Encapsulation (MPE) was considered for both forward- and return-links.

The configuration of the baseline scenario was modified in order to assess the performance of ACM on the forward-link. First FMT techniques were activated so that adaptive coding and modulation was available on the forward-link. The encapsulation / decapsulation scheme was also modified: the Generic Stream Encapsulation (GSE) was selected. The scheduler type in the forward-link was also modified so that it optimizes the selection of packets according to the quality of service and the ModCod. The number of terminals was adjusted after several simulations / computations so that the FL is still in overload situation even if the capacity is 
increased because of ACM. The return-link was dimensioned so that it does not penalize the forward-link (overdimensioning via the number of carriers).

The performance of ACM on the return-link was assessed exactly the same way as on the forward-link. The configuration of the reference scenario (baseline) was modified to introduce new mechanisms / parameters. ACM was switched on in the return-link, GSE was selected, the Fixed Burst Length format (400/800) was chosen and the number of terminals was increased to stay in overload conditions. As earlier the forward-link was over-dimensioned in order to have no influence on the RL measurements.

The simulation duration was fixed to 2 hours for the three scenarios: baseline, ACM on FL and ACM on RL. The measurement interval was set to 10 seconds. It has to be specified that rain statistics for generating the time series (rain fading events) are issued from average year statistics.

\subsection{Forward-Link}

The way the forward-link is loaded depends directly on the number of satellite terminals that are simulated. The traffic generators involve the transmission of data, more particularly packets arrive into the pools located in the gateway scheduler before being sent on the forward-link. The analysis of the total input and output bit rates in the gateway scheduler permits to get an estimation on how the forward-link reacts during the simulation. The total input and output bit rates contain IP packets but signalling data too.

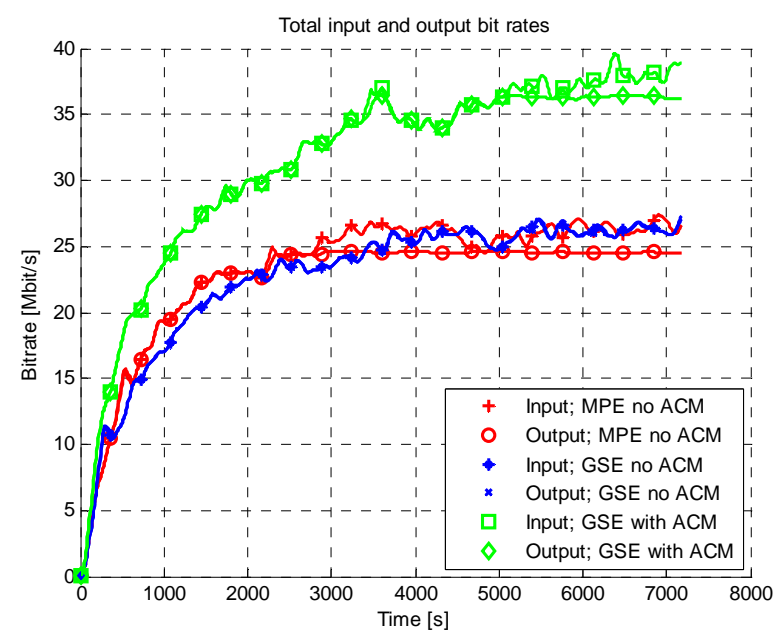

Figure 9: Gateway Scheduler - Total I/O bit rates.

While observing the previous figure (Figure 9) several remarks can be drawn. Firstly there is a "transient" period at the beginning of each curve (and this will be mainly the case in all other results). This "transient" period is related to the traffic generators which all start at the same time, i.e. at the very beginning of the simulation, and need to have simulated at least one session. The stability is reached after about one hour of simulation (at $t>3600 s$ ). Secondly there is a clear difference between the three scenarios. The non-adaptive scenario, i.e. the one without ACM (CCM) and MPE, shows that the output bit rate saturates after a while. The quantity of traffic at the input of the scheduler is higher than the output one. This means the system cannot transmit all data since it is in overload situation. The non-adaptive scenario (no ACM) but using GSE (simple modification of configuration) reveals that GSE performs better than MPE. The output bit rate curve follows the input rate meaning that the system is not saturated anymore just by using another encapsulation scheme. This is related to the higher efficiency of GSE (less overhead, better fragmentation). Finally the scenario in which both ACM and GSE are used shows impressive results. The saturation of the system is also reached but to do so the number of satellite terminals had to be significantly increased. This results into a higher effective output bit rate in the gateway scheduler.

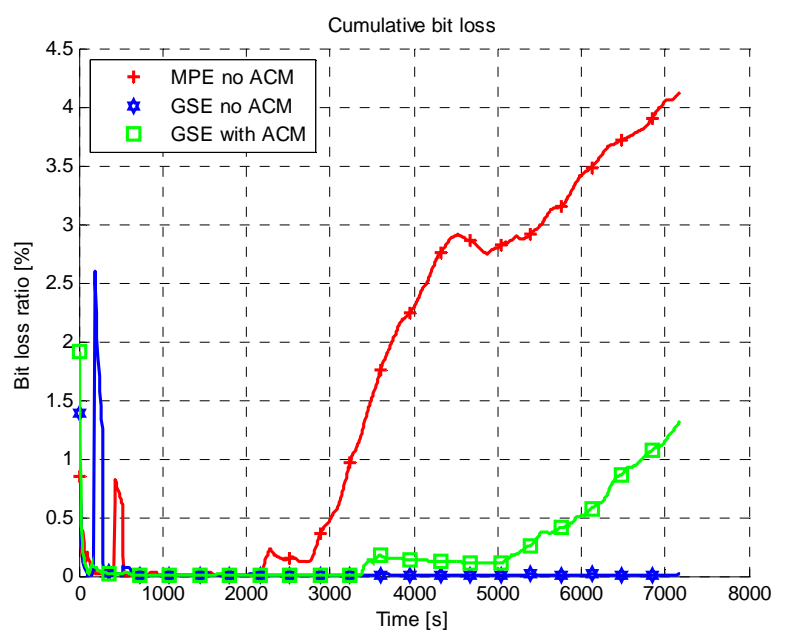

Figure 10: Gateway Scheduler - Total cumulative bit loss.

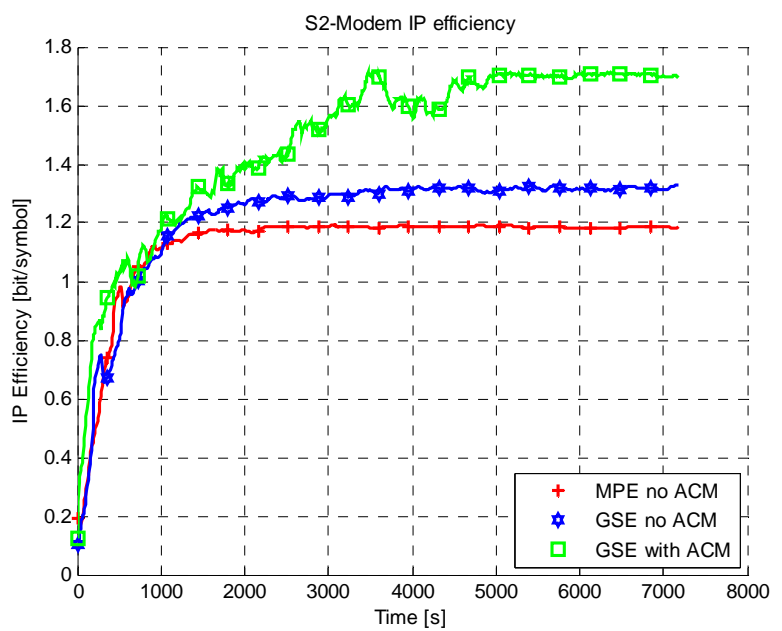

Figure 11: Gateway S2 Modem - IP efficiency.

Figure 10 illustrates the total cumulative bit loss in the gateway scheduler. Actually the scheduler monitors the number of bits lost in the different pools. When packets are staying too long in the pools there are dropped according to a timeout parameter (which is pool-dependant). The figure shows that in the first scenario bits are lost since the system is saturated. Since the input bit rate is getting too high the system has to drop packets from the pools. The utilization of GSE solves the problem: bits are not lost because the system 
is no more overloaded. The last scenario shows some bit losses: it was configured to reach an overload state too.

In Figure 11 the analysis of the measurements was performed in the gateway S2 modem. This module receives the BBFRAMES containing encapsulated data (MPEG-TS packets or GSE packets). This module is aware of the number of bits at IP level (additional information available at this level) that are inserted on the carrier. This gives an IP efficiency which is expressed in bits per symbol. The IP efficiency is thus the number of IP bits carrying data from applications over the total number of symbols at physical layer. This measure is strongly related to the coding and modulation. The curves show the IP efficiency obtained for each scenario. Using GSE over MPE already increases the IP efficiency by about $10 \%$ (reduction of the overhead compared to MPEG-TS) but enabling ACM boosts the overall efficiency of the forward-link.

Figure 12 represents the ModCod distribution in the forwardlink. Actually 19 ModCods out of the 28 ModCods available are utilized. Some ModCods were not considered since their critical thresholds overlap other ModCods. The figure shows which ModCods are used and in which proportion. QPSK 1/4 (on the left) is mainly used for signalling (most robust ModCod).

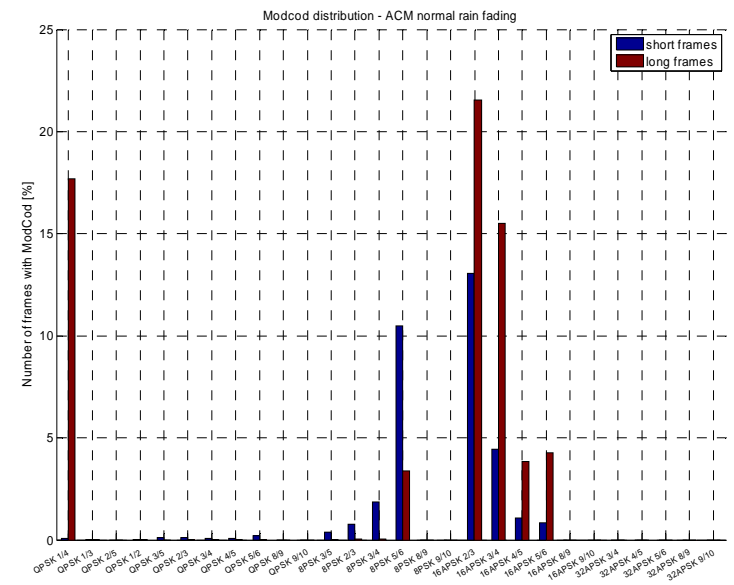

Figure 12: Forward-link ModCod distribution.

The table below (Table 1) summarizes the results shown for the three different scenarios considered: MPE without ACM, GSE without ACM and GSE with ACM.

\begin{tabular}{|l|c|c|c|c|}
\hline Measure & Unit & $\begin{array}{c}\text { MPE no } \\
\text { ACM }\end{array}$ & $\begin{array}{c}\text { GSE no } \\
\text { ACM }\end{array}$ & $\begin{array}{c}\text { GSE } \\
\text { ACM }\end{array}$ \\
\hline $\begin{array}{l}\text { Input bit } \\
\text { rate }\end{array}$ & Mbps & 26.3 & 27.2 & 37.6 \\
\hline $\begin{array}{l}\text { Output bit } \\
\text { rate }\end{array}$ & Mbps & 24.5 & 27.2 & 36.3 \\
\hline Loss bit rate & $\%$ & 6.6 & 0.0 & 3.4 \\
\hline $\begin{array}{l}\text { IP } \\
\text { efficiency }\end{array}$ & bit/sym & 1.19 & 1.32 & 1.70 \\
\hline
\end{tabular}

Table 1: Summary of the results obtained in the forward-link. The table above (Table 1) shows the benefit of selecting the Generic Stream Encapsulation (GSE) and also of using adaptive coding and modulation (ACM) in the forward-link. The gain in terms of IP efficiency for using GSE instead of MPE is about $11 \%$ (the output bit rate cannot be considered since the system was not overloaded anymore). Using both GSE and ACM in the FL involves an increase of the capacity (output bit rate) of about $48 \%$ and an increase of the IP efficiency of about $43 \%$. Note that the total number of satellite terminals configured for GSE with ACM was increased to 782 so that the system was loaded in the same conditions. This results into an increase of the number of satellite terminals of about $40 \%$.

\subsection{Return-Link}

The results in the return-link are obtained differently. In the forward-link the measurements were directly performed in the gateway (the gateway takes care of the transmission on the forward-link). On the contrary in the return-link the measurements are performed by the satellite terminals themselves. Since there are different types of satellite terminals (SOHO, SME1 and SME2 / Gold and Silver) measurements were aggregated among the terminals of the same type. Some measurements are thus related to a particular terminal type (all SOHO Gold and Silver satellite terminals for instance).

As previously the transmission of the data on the return-link is performed by the terminal scheduler and the encapsulation / fragmentation MAC modules present in each satellite terminal.

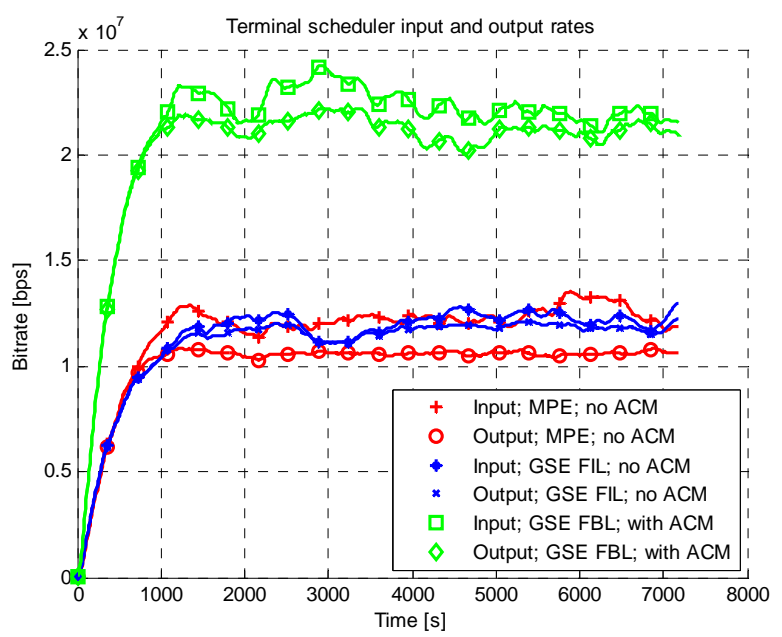

Figure 13: Terminal scheduler - Total I/O bit rates.

The previous figure (Figure 13) shows the total input and output bit rates of the terminal schedulers (all terminal types considered). The same observations as the ones realized for the forward-link can be done. The output bit rate is below the input rate for the scenario using MPE without ACM. This is related to the fact the system was dimensioned in such a way that it was in overload situation. Using GSE instead of MPE already causes the system not to be saturated anymore. As before GSE performs better than MPE by reducing the overall overhead and by increasing the efficiency. Finally when 
ACM is enabled one can see the impressive increase of capacity even if the system is still overloaded.

The two next figures (Figure 14 and Figure 15) illustrate the physical layer (PHY) efficiency respectively for SOHO and SME2 satellite terminal types. Such a measurement has to be considered separately since the transmission capacity, and as consequence the efficiency, depend directly on the satellite terminal type (different link budgets). For both SOHO and SME2 terminals the efficiency increases dramatically while changing the encapsulation scheme (GSE) and enabling ACM.

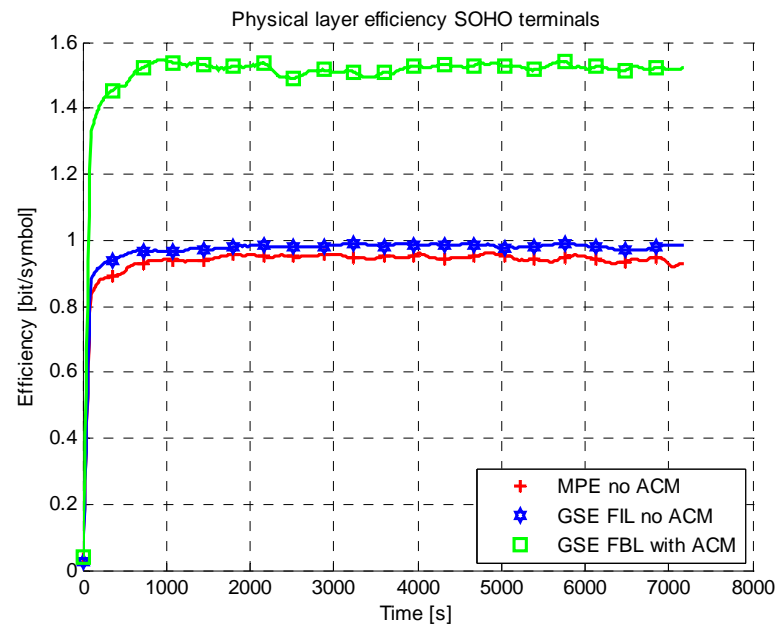

Figure 14: SOHO terminal modem - PHY efficiency.

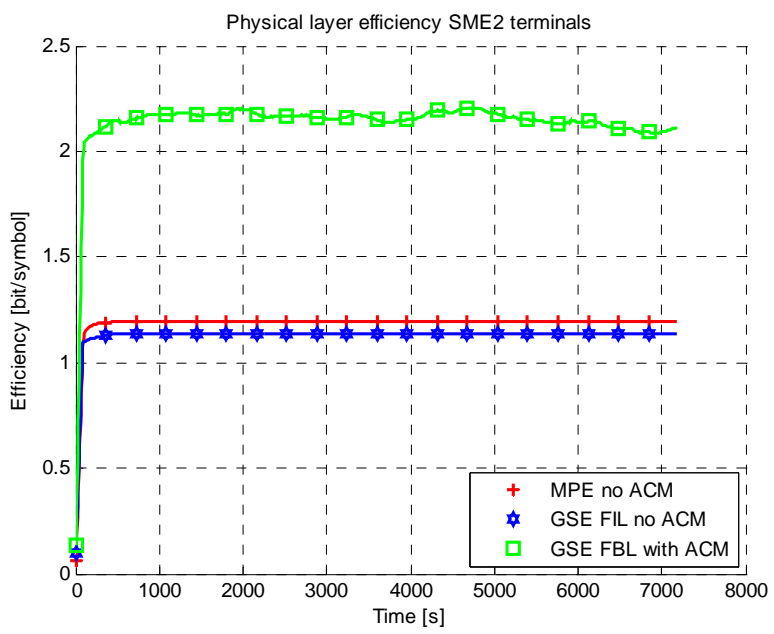

Figure 15: SME2 terminal modem - PHY efficiency.

The last figure (Figure 16) shows the ModCod distribution in the return-link. In this case 18 ModCods are used. On can see that more efficient ModCods are utilized compared to the CCM case (mainly 8PSK ModCods). This leads to an increase of the physical layer efficiency thus to an increase of the IP efficiency (not shown here).

The different results obtained while assessing the performance of GSE and ACM in the return-link are summarized in the next table (Table 2).

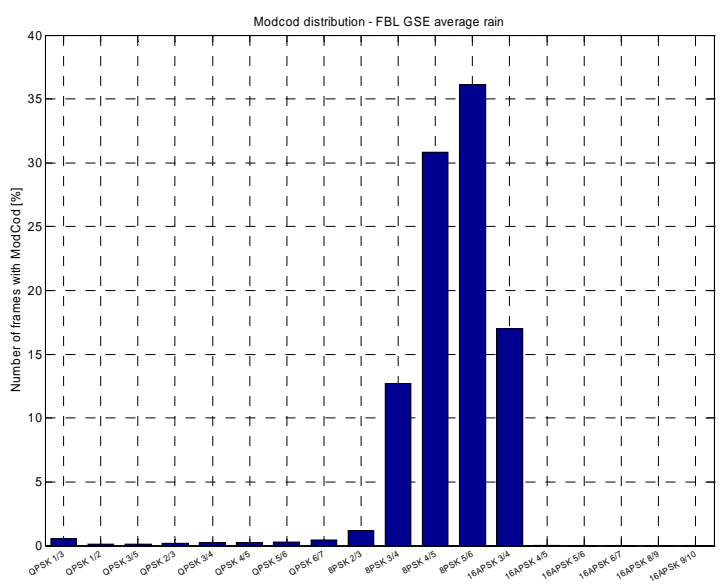

Figure 16: Return-link ModCod distribution.

\begin{tabular}{|l|c|c|c|c|}
\hline Measure & Unit & $\begin{array}{c}\text { MPE no } \\
\text { ACM }\end{array}$ & $\begin{array}{c}\text { GSE no } \\
\text { ACM }\end{array}$ & $\begin{array}{c}\text { GSE } \\
\text { ACM }\end{array}$ \\
\hline $\begin{array}{l}\text { Input bit } \\
\text { rate }\end{array}$ & Mbps & 12.5 & 12.3 & 22.0 \\
\hline $\begin{array}{l}\text { Output bit } \\
\text { rate }\end{array}$ & Mbps & 10.6 & 11.9 & 21.0 \\
\hline $\begin{array}{l}\text { SOHO PHY } \\
\text { efficiency }\end{array}$ & bit/sym & 0.95 & 0.98 & 1.53 \\
\hline $\begin{array}{l}\text { SME1 PHY } \\
\text { efficiency }\end{array}$ & bit/sym & 1.18 & 1.12 & 2.07 \\
\hline $\begin{array}{l}\text { SME2 PHY } \\
\text { efficiency }\end{array}$ & bit/sym & 1.20 & 1.14 & 2.15 \\
\hline
\end{tabular}

Table 2: Summary of the results obtained in the return-link.

Using GSE instead of MPE allows an increase of capacity of about $12 \%$ when analysing the output bit rate of the terminal schedulers. Using both GSE and ACM permits to reach higher bandwidth in identical load situations: about $98 \%$ of gain. The physical efficiency depends on the individual terminal type link budget which determines the usable ModCods. For this reason the performances of GSE and ACM are different: about $61 \%$ for the SOHO terminals, about $75 \%$ for the SME1 terminals and about $79 \%$ for the SME2 terminals. In the scenario using GSE and ACM the total number of satellite terminals was fixed to 1072 so that the load situation remains the same. This corresponds to an increase of the number of satellite terminals of about $91 \%$.

\section{Conclusions}

In this paper the performances of adaptive coding and modulation (ACM) in DVB-S2/advanced DVB-RCS satellite systems were assessed. The different measurements were performed by using a complete advanced simulator system. The results show impressive gain on both forward- and return-links. With the configuration considered the capacity increases by about $48 \%$ and $98 \%$ respectively in the forwardlink and in the return-link. This is achieved by getting higher spectral efficiency via the coding and modulation modes but also by selecting another encapsulation scheme: the Generic Stream Encapsulation (GSE). 


\section{Acknowledgements}

The satellite system simulator presented in this paper was developed in the framework of the ESA funded project "Resources Management using Adaptive Fade Mitigation Techniques (FMT) in DVB-RCS Multi-Beam Systems”. ESA Study 18826/05/NL/US, 2005. The consortium consisted of TriaGnoSys GmbH (project leader), Audens ACT, German Aerospace Center (DLR), and NERA.

\section{References}

[1] G. Maral, M. Bousquet. "Satellite Communications Systems”, Fourth Edition, (2002).

[2] E. Lutz, M. Werner, A. Jahn. "Satellite Systems for Personal and Broadband Communications”, (2000).

[3] R. Rinaldo, R. De Gaudenzi. "Capacity analysis and system optimization for the forward link of multi-beam satellite broadband systems exploiting adaptive coding and modulation", International Journal of Satellite Communications and Networking, vol. 22, pp. 401-423, issue 3, (June 2004).

[4] R. De Gaudenzi, R. Rinaldo. "Adaptive Coding and Modulation for Next Generation Broadband Multimedia Systems", AIAA-2002-1863, (2002).

[5] Digital Video Broadcasting (DVB): "Second generation framing structure, channel coding and modulation systems for broadcasting, interactive services, new gathering and other broadband satellite applications”, European Standard (Telecommunications series), ETSI EN 302307 V1.1.2, (2006-06).

[6] Digital Video Broadcasting (DVB): "Interaction channel for satellite distribution systems”, European Standard (Telecommunication series), ETSI EN 301790 V1.5.1, (2009-01).

[7] H. Brandt, V. Boussemart, C. Kissling, C. Párraga Niebla, O. Lücke, R. Vilalta, E. Dupont, R. Schweikert, T. Wörz. "Resources Management using Adaptive Fade Mitigation Techniques in DVB-S2/DVB-RCS MultiBeam Systems", Proceedings of the International Communication Satellite Systems Conference (ICSSC'07), (2007).

[8] T. Wörz, R. Schweikert, A. Jahn, R. Rinaldo. "Physical layer efficiency of satellite DVB using fade mitigation techniques", Proceedings of the International Communication Satellite Systems Conference (ICSSC'05), Rome, Italy, (September 2005).

[9] A. Varga. "The OMNET++ Discrete Event Simulation System", Proceedings of the European Simulation Multiconference (ESM'2001), Prague, Czech Republic, (June 6-9, 2001).

[10] V. Boussemart, O. Grémillet. "Investigation on the SelfSimilarity of Web Traffic Generated by Aggregated Individual Browsers", Proceedings of the International Communication Satellite Systems Conference (ICSSC'05), Rome, Italy, (September 2005).

[11] C. Párraga Niebla. "Scheduling Techniques for Satellite Systems with Adaptive Coding and Modulation",
Proceedings of ASMS Conference, Herrsching, Germany, (May 29-31, 2006). 